\title{
Construction of Fuzzy Map for Autonomous Mobile Robots Based on Fuzzy Confidence Model
}

\author{
Jung-Fu Hou, ${ }^{1}$ Yau-Zen Chang, ${ }^{1}$ Ming-Hsi Hsu, ${ }^{1}$ Shih-Tseng Lee, ${ }^{2}$ and Chieh-Tsai Wu ${ }^{2}$ \\ ${ }^{1}$ Department of Mechanical Engineering, Chang Gung University, Taoyuan 33302, Taiwan \\ ${ }^{2}$ Department of Neurosurgery, Chang Gung Memorial Hospital, Taoyuan 33305, Taiwan \\ Correspondence should be addressed to Yau-Zen Chang; zen@mail.cgu.edu.tw
}

Received 15 January 2014; Revised 23 April 2014; Accepted 25 April 2014; Published 16 June 2014

Academic Editor: Leo Chen

Copyright (c) 2014 Jung-Fu Hou et al. This is an open access article distributed under the Creative Commons Attribution License, which permits unrestricted use, distribution, and reproduction in any medium, provided the original work is properly cited.

\begin{abstract}
This paper presents the use of fuzzy models to explicitly consider sensor uncertainty and finite resolution in solving the SLAM (simultaneous localization and mapping) problem for autonomous mobile robots. The approach establishes fuzzy confidence models in describing occupied obstacles and available space. The problem is transformed into an optimization task of minimizing the alignment error between newly scanned local fuzzy maps and selected parts of a developing global fuzzy map. In aligning local fuzzy maps into a global fuzzy map, we developed a prediction strategy to crop the most potential part from the sensed local fuzzy maps to be overlapped with the global fuzzy map. A mobile vehicle equipped with a laser range finder, the Hokuyo URG-04LX, is used to demonstrate the procedure of fuzzy map building. Experimental results show that the proposed architecture is effective in generating a comprehensive global fuzzy map, which is suitable for both human comprehension and path design during real-time navigation.
\end{abstract}

\section{Introduction}

An essential task of an autonomous mobile robot is to determine its location and construct a map of its environment, usually denoted as the work of solving the SLAM (simultaneous localization and mapping) problem [1-3]. Selflocalization is about finding the location of a robot in a map, while mapping is about constructing a referable map when the robot is moving in an unknown or changing environment.

Autonomous map construction has been under extensive research for decades [4-9]. For instance, Chong and Kleeman [5] used a sonar sensor and a positioning sensor, and Jaradat and Langari [6] used a sonar sensor in developing the OGM (occupancy grid map) method, where the environment is simplified into occupied and vacant grids. Guivant et al. [7] used encoders in cooperation with a laser range finder for positioning. Davison and Kita [8] combined an accelerometer and two dynamic video cameras to construct irregular maps. Tomono [9] used baselines as the basis for a video camera to choose the characteristic points for map reconstruction.

There are various kinds of sensors developed for these tasks, such as sonar [10,11], laser range finders [12], and video cameras [13]. Sonar is effective in detecting range, but only a narrow region can be detected at one time. Laser range finders can effectively provide $2 \mathrm{D}$ environmental information at high refresh rate, up to 10 frames per sec, but may fail to sense black objects and complex 3D obstacles. Moreover, video cameras can emulate the capability of human eyes, but huge computing power is required for real-time implementation.

An early work [14] proposed a fuzzy model for the sonar sensing, but the paper lacks detailed procedures for the SLAM problem. Inspired by the research, this paper presents the use of a fuzzy model to explicitly consider sensor uncertainty and finite resolution of laser range finders in solving the SLAM problem.

Our proposed system is realized by establishing a fuzzy confidence model, which is composed of sensed obstacles and assured space based on sensor readings. The SLAM problem is transformed into an optimization task of minimizing the alignment error between newly scanned local fuzzy maps and selected parts of a developing global fuzzy map. The task is then solved by the Cuckoo search optimization algorithm $[15,16]$. Being a nature-inspired meta-heuristic algorithm, 


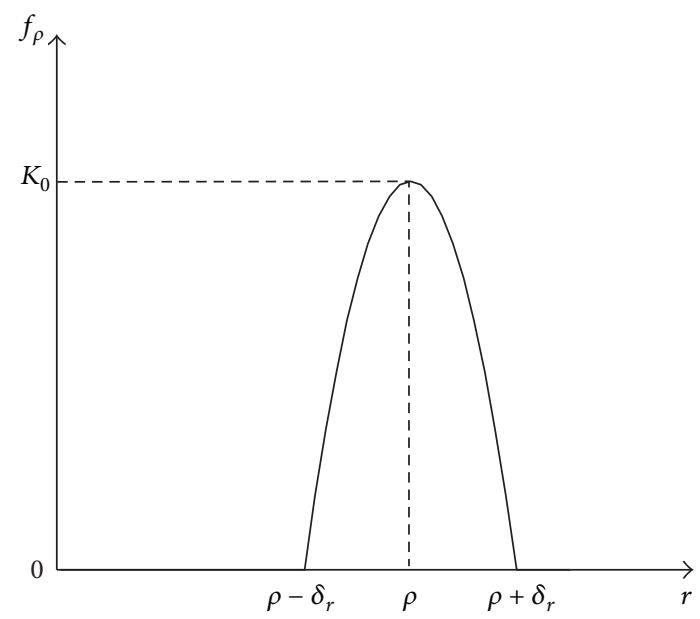

Figure 1: The profile of a confidence function $f_{\rho}$ which varies around a given range reading $\rho$.

the algorithm can efficiently provide a set of optimal solutions within a reasonable number of cost function evaluations.

To ease the search, a prediction strategy is applied such that only the most promising portions of the newly scanned local fuzzy maps and last fuzzy maps are involved in the registration. The search starts with adequate knowledge of moving direction, which makes essentially no assumption about the environment and is able to incrementally build a global map in real time. A specific laser range finder, the Hokuyo URG-04LX, is used to demonstrate the use of the confidence model in the building of fuzzy maps.

\section{Certainty Model of the Sensor}

2.1. Characteristics of the Laser Range Finder. Most distance sensors, such as laser range finders, suffer from both measurement error and finite resolution, which introduce uncertainties deteriorating with distance. Knowledge about these uncertainties may be described explicitly by fuzzy membership functions in order to build confidence models.

To model the knowledge of measurement error in terms of the degree of certainty, confidence level for the existence of an obstacle can be described as a function symmetric to a given range reading $\rho$. Assuming that $K_{0}$ is a parameter corresponding to the maximum confidence level, the confidence function, $f_{\rho}$, can be described as [14]

$$
f_{\rho}(r)= \begin{cases}K_{0} \cdot\left[1-\left(\frac{r-\rho}{\delta_{r}}\right)^{2}\right], & \text { for }|r-\rho| \leq \delta_{r}, \\ 0, & \text { otherwise. }\end{cases}
$$

The profile of $f_{\rho}$ is depicted in Figure 1, where $\delta_{r}$ is the bound of measurement error. According to the figure, the confidence level decreases along a parabolic shaped trajectory toward zero as the distance to the range reading $\rho$ increases.

For a typical distance sensor, the effects of finite lateral resolution can be formulated into another confidence function similar to (1). Hence, we create a hybrid function $B_{\rho, \phi}$ to represent the level of confidence for the combination of measurement error and finite resolution as

$$
\begin{aligned}
& B_{\rho, \phi}(r, \theta) \\
& = \begin{cases}K_{r \theta} \cdot\left[1-\left(\frac{r-\rho}{\delta_{r}}\right)^{2}\right] \\
\times\left[1-\left(\frac{\theta-\phi}{\delta_{\theta}}\right)^{2}\right], & \text { when }|r-\rho| \leq \delta_{r},|\theta-\phi| \leq \delta_{\theta}, \\
0, & \text { otherwise. }\end{cases}
\end{aligned}
$$

As depicted in Figure 2, the profile of the confidence level $B_{\rho, \phi}$ is in the polar coordinate system $(r, \theta)$, which is symmetric to both the range reading $\rho$ and angular reading $\phi$, where $\delta_{r}$ is the bound of longitudinal distance error and $\delta_{\phi}$ is the bound of finite lateral resolution. The confidence level decreases toward zero as the distance to the range reading $\rho$ or angular reading $\phi$ increases.

Information about the existence of obstacles is crucial for the construction for maps, providing valuable environment knowledge to the human supervisor. On the other hand, the information of free space between the sensor and the obstacles is vital for navigation of moving robots, since sensors cannot provide information behind obstacles. The confidence function which describes the availability of free space for navigation is defined as a function $P_{\rho, \phi}(r, \theta)$ corresponding to a set of range reading $\rho$ and angular reading $\phi$ in the polar coordinate system:

$$
\begin{aligned}
& P_{\rho, \phi}(r, \theta) \\
& = \begin{cases}\left(\frac{r-\rho}{\delta_{r}}\right)^{2} \\
{\left[1-\left(\frac{\theta-\varphi}{\delta_{\theta}}\right)^{2}\right],} & \text { when } \rho-\delta_{r} \leq r \leq \rho,|\theta-\phi| \leq \delta_{\theta}, \\
1-\left(\frac{\theta-\varphi}{\delta_{\theta}}\right)^{2}, & \text { when } r<\rho-\delta_{r},|\theta-\phi| \leq \delta_{\theta}, \\
0, & \text { otherwise. }\end{cases}
\end{aligned}
$$

The profile of $P_{r, \theta}$ is depicted in Figure 3. We have that the $3 \mathrm{D}$ shape is the opposite to that of Figure 2. According to the figure, we have full confidence about the available space between the observer and a measured obstacle located at $(\rho, \phi)$. On the contrary, we have little confidence about the space behind the sensed obstacle.

2.2. Certainty Model of a Specific Laser Range Finder. The Hokuyo URG-04LX is a popular laser range finder (LRF) which uses a $785 \mathrm{~nm}$ semiconductor laser beam. It has a fixed scanning range of 60 to $300^{\circ}$ with a $0.36^{\circ}$ angular resolution and a $100 \mathrm{msec}$ scan rate. The data transfer rate can be set at 9 Mbps when connected via USB.

The LRF has a quoted range of effective measurement between 20 and $4,095 \mathrm{~mm}$. The measurement error is $\pm 10 \mathrm{~mm}$ for distances of less than $1 \mathrm{~m}$. For greater distances, the error is $\pm 1 \%$ of the range readings $[17,18]$. The variation of 


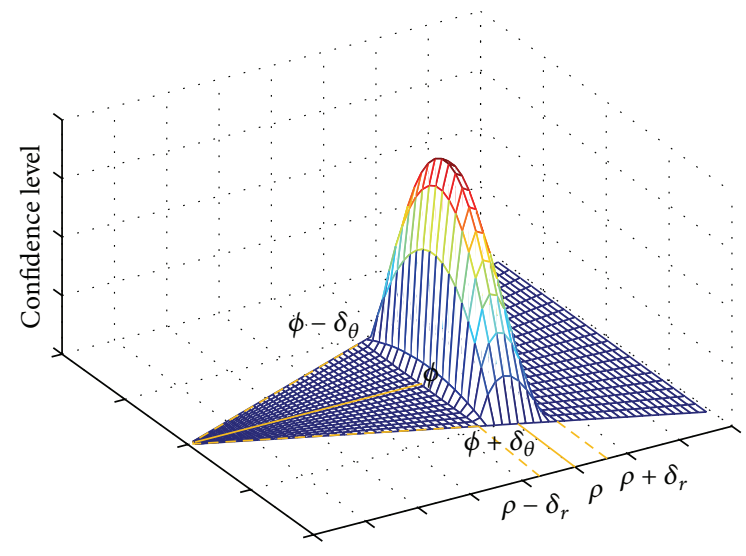

FIGURE 2: The confidence function $B_{\rho, \phi}$ of a distance sensor combines the effects of measurement error and finite resolution around a set of range reading $\rho$ and angular reading $\phi$.

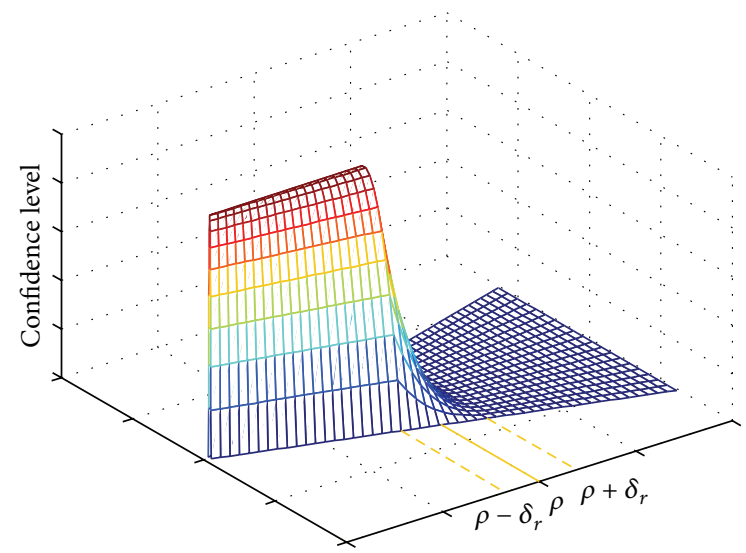

Figure 3: The confidence function $P_{r, \theta}$ represents the confidence in the availability of space based on range reading $\rho$ and angular reading $\phi$.

measurement error in terms of measured distance is depicted in Figure 4. As confidence in measured values decreases with the increase of measurement error, the complementary curve to the measurement error curve is defined as the confidence curve and illustrated in the figure, which can be fitted by a 6 th order polynomial function:

$$
\begin{aligned}
K_{r \theta}(r)= & 4.70 \times 10^{-21} \times r^{6}-5.96 \times 10^{-17} \times r^{5}+2.82 \\
& \times 10^{-13} \times r^{4}-5.96 \times 10^{-10} \times r^{3}+4.56 \\
& \times 10^{-7} \times r^{2}-1.12 \times 10^{-4} \times r+0.76 .
\end{aligned}
$$

The confidence level, which is a function of range reading as shown in Figure 4, is used as the parameter $K_{r \theta}$ in (2) with $\delta_{\theta}$ being $0.36^{\circ}$. In order to further clarify how the confidence levels vary with distance measure, 5 confidence levels with respect to corresponding obstacle detections are demonstrated in Figure 5, where the dotted green curve is generated by the closed form of (4).

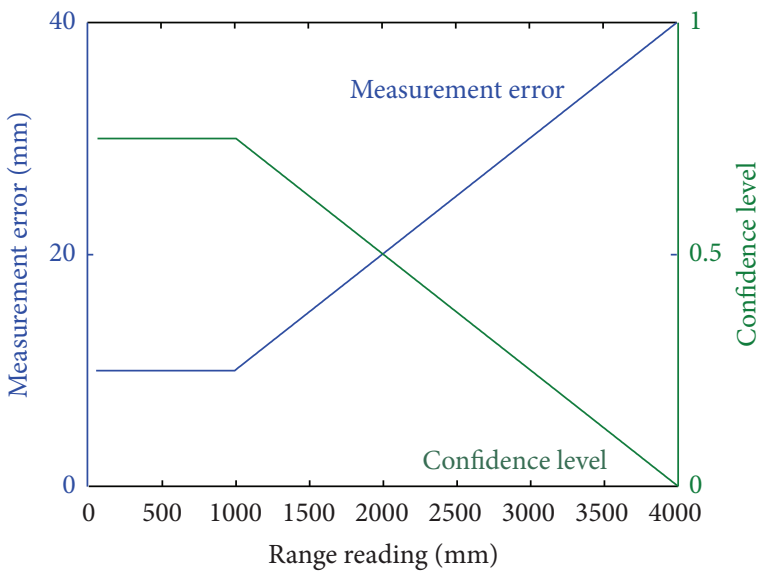

FIGURE 4: Graphic illustration of measurement error as a function of the range reading. The complementary curve is used as the confidence function.

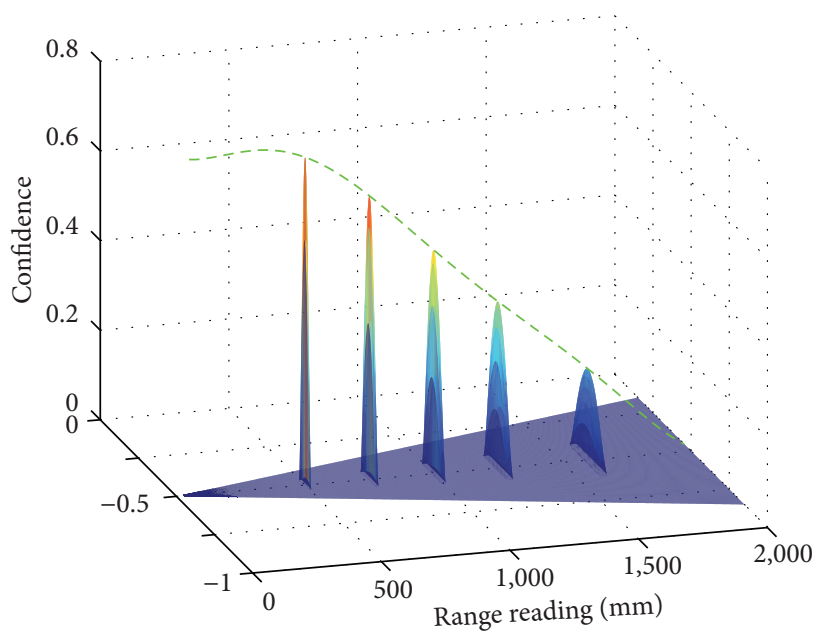

Figure 5: Profile of confidence functions corresponding to different range readings.

2.3. Aggregation for Fuzzy Map Construction. The construction of a local fuzzy map of obstacles at time $t$, denoted as $M_{o}(t)$, is based on the aggregation of several sets of range reading and angular reading, $\left(\rho_{k}, \theta_{k}\right)$, in polar coordinates:

$$
\mu_{\text {Occupied }}=M_{o}(t)=\bigcup_{k} B_{r \theta}\left(\rho_{k}(t), \phi_{k}(t)\right) .
$$

Similarly, a local fuzzy map of space at time $t$, denoted as $M_{s}(t)$, is based on the aggregation of several sets of range reading and angular reading, $\left(\rho_{k}, \theta_{k}\right)$, in polar coordinates:

$$
\mu_{\text {Space }}=M_{s}(t)=\bigcup_{k} P_{r \theta}\left(\rho_{k}(t), \phi_{k}(t)\right) .
$$




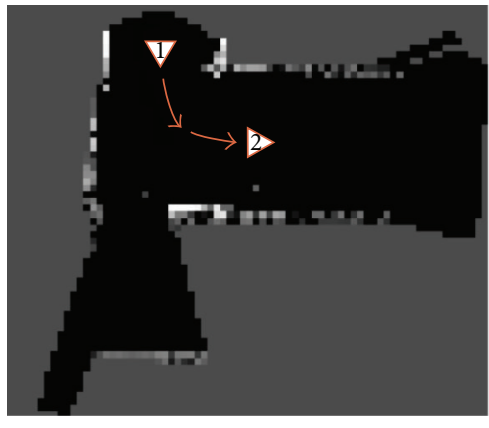

(a) 2D fuzzy map

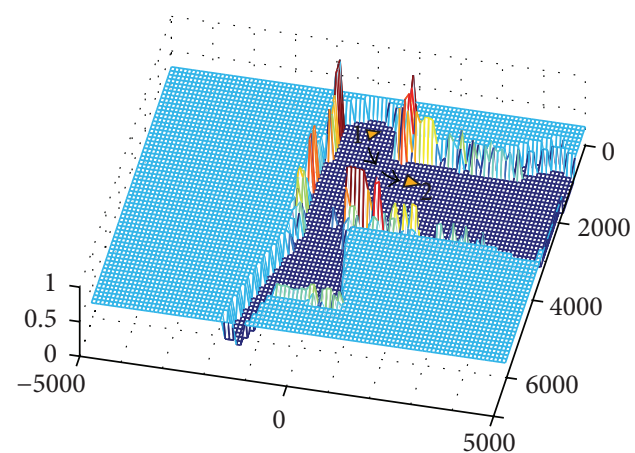

(b) 3D fuzzy map

FIGURE 6: Illustration of fuzzy maps showing both the occupied obstacles and available space. The space is in the darkest color in the diagrams.

Furthermore, to construct a fuzzy map simultaneously suitable for human comprehension and path design, the occupied obstacles and available space should be integrated together:

$$
\begin{aligned}
\mu_{\text {Map }} & =M_{\text {all }}(t)=\left(0.3 \cup M_{o}(t)\right) \cap \overline{M_{s}}(t) \\
& =\min \left(\max \left(0.3, M_{o}(t)\right), \overline{M_{s}}(t)\right),
\end{aligned}
$$

where $\overline{M_{s}}(t)$ is the complement of $M_{s}(t)$ and the value 0.3 is selected to distinguish uncertain regions from assured occupied and space regions.

A practical implementation of (7) is demonstrated in Figure 6 where the triangles marked with 1 and 2 are the initial and final positions, respectively, of a moving robot carrying a laser range finder. Figures 6(a) and 6(b) are two-dimensional diagram and three-dimensional diagram, respectively. Note that the space is in the darkest color showing the least level, which is suitable for path planning. The largest areas with gray value, assigned as 0.3 in this example, are the regions behind sensed obstacles and hence unknown to the sensor.

\section{Experimental Study}

3.1. Registration between Local Fuzzy Maps. The concept of registration is based on the similarities between two local fuzzy maps sensed at different instances. As a robot moves, the scene changes with the emergence and disappearance of objects and boundaries. These extra items on the maps increase the difficulty in the judge of matching between two succeeding local maps, leading to erroneous results. To alleviate the difficulty and enhance robustness and correctness in the search of coordinate transformation for alignment, a prediction algorithm is proposed. The algorithm exploits the knowledge of current move direction to crop local maps from the sensed maps.

Let $\left.M_{o}(t)\right|_{(x, y)}$ and $\left.M_{o}(t+1)\right|_{(x, y)}$ be the local fuzzy maps sensed at $t$ and $t+1$ which have been transformed into rectangular coordinates from their polar coordinate counterparts, $M_{o}(t)$ and $M_{o}(t+1)$, respectively. Besides, let $p_{k}(t)$

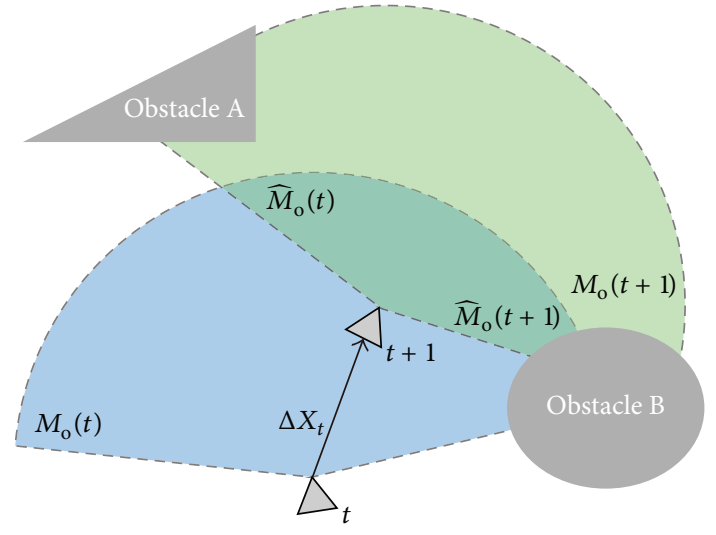

FIGURE 7: The cropping of local maps for registration by the use of estimated motion.

and $p_{j}(t+1)$ be position vectors with confidence values at $\left.M_{o}(t)\right|_{(x, y)}$ and $\left.M_{o}(t+1)\right|_{(x, y)}$, respectively. The problem is to find coordinate transformations, ${ }_{t}^{t+1} T\left(\Delta X_{t}\right)$ and ${ }_{t+1}^{t} T\left(\Delta X_{t}\right)$, such that the fuzzy maps are aligned with each other. Here $\Delta X_{t}$ is a vector of displacement and rotation for the robot to move between $t$ and $t+1$. To begin with the prediction procedure, a rough estimation of $\Delta X_{t}$, denoted as $\Delta \overline{X_{t}}$, is generated based on current moving command. Partial local fuzzy maps, $\left.\widehat{M}_{o}(t+1)\right|_{(x, y)}$ and $\left.\widehat{M}_{o}(t)\right|_{(x, y)}$, that are cropped from $M_{o}(t+1)$ and $M_{o}(t)$, are defined as

$$
\begin{aligned}
\left.\widehat{M}_{o}(t+1)\right|_{(x, y)}= & \left\{\begin{array}{l}
t+1 \\
t
\end{array}\left(\Delta \overline{X_{t}}\right) p_{k}(t)\left|p_{k}(t) \in M_{o}(t)\right|_{(x, y)}\right\} \\
\cap & \left.M_{o}(t+1)\right|_{(x, y)}, \\
\left.\widehat{M}_{o}(t)\right|_{(x, y)}= & \left\{\begin{array}{l}
t+1 \\
t
\end{array}\left(\Delta \overline{X_{t}}\right) p_{j}(t+1) \mid\right. \\
& \left.\left.p_{j}(t+1) \in M_{o}(t+1)\right|_{(x, y)}\right\}\left.\cap M_{o}(t)\right|_{(x, y)} .
\end{aligned}
$$

The relationship between the local fuzzy maps is illustrated in Figure 7. Besides, the homogeneous transformation 


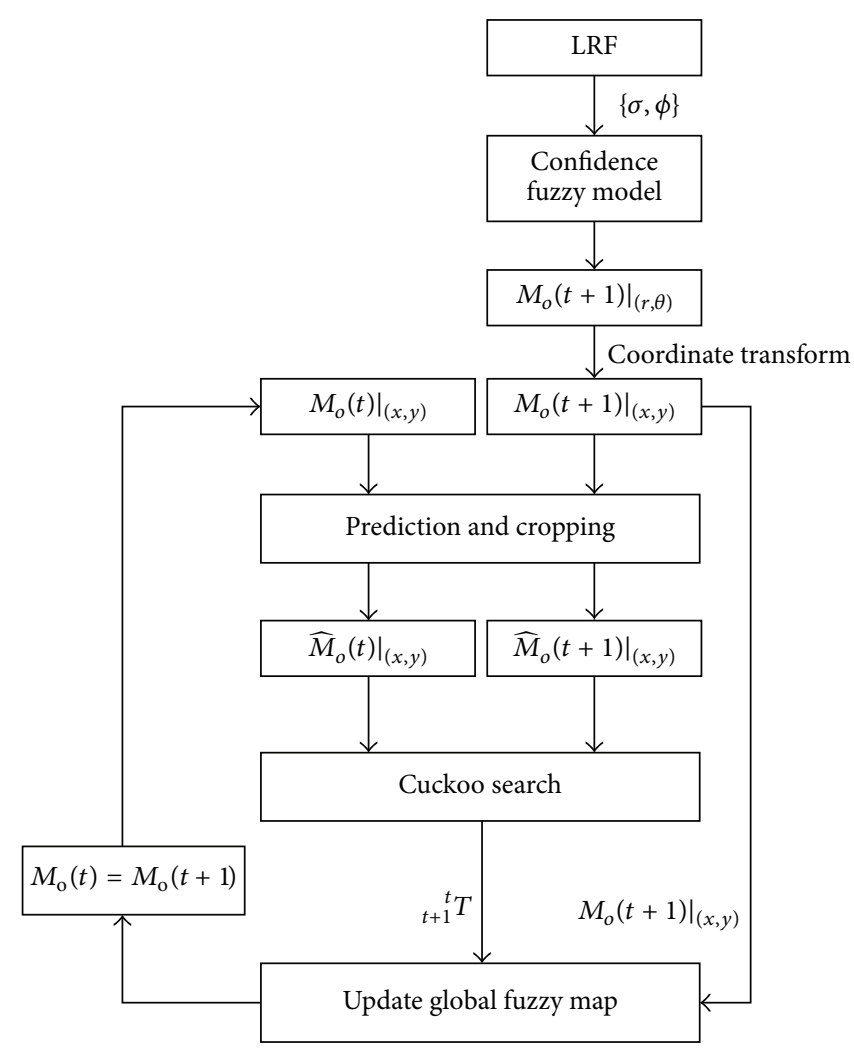

FIgURE 8: Flowchart of the generation of a global fuzzy map.

matrices, ${ }_{t}^{t+1} T\left(\Delta \bar{X}_{t}\right)$ and ${ }_{t+1}^{t} T\left(\Delta \bar{X}_{t}\right)$, can be easily obtained by exploiting the relationship ${ }_{t}^{t+1} T\left(\Delta \bar{X}_{t}\right)={ }_{t+1}^{t} T\left(\Delta \bar{X}_{t}\right)^{-1}=$ ${ }_{t+1}^{t} T\left(\Delta \bar{X}_{t}\right)^{T}$.

3.2. System Flowchart. This section presents an alternative solution to simultaneous localization and mapping (SLAM) problem by direct registration of fuzzy maps of obstacles.

As shown in the flowchart of Figure 8, the data $\{\rho, \phi\}$ sensed by the laser range finder are firstly aggregated into a local fuzzy map $M_{o}(t+1)$ in polar coordinates and transformed into rectangular coordinate system, denoted as $\left.M_{o}(t+1)\right|_{(x, y)}$. The prediction algorithm of (8) and (9) is then used to crop from this local fuzzy map $\left.M_{o}(t)\right|_{(x, y)}$ and the last local fuzzy map $\left.M_{o}(t+1)\right|_{(x, y)}$ to generate two fuzzy maps, $\left.\widehat{M}_{o}(t+1)\right|_{(x, y)}$ and $\left.\widehat{M}_{o}(t)\right|_{(x, y)}$, for registration by the Cuckoo search [14], where a cost function of overlap between them is maximized. The registration results in a homogeneous transformation matrix ${ }_{t+1}^{t} T$ to be used for the local fuzzy map $M_{o}(t+1)$ to be integrated into the global fuzzy map in real time. The procedure iterates with the movement of the robot and the receiving of sensed data.

The data $\{\rho, \varphi\}$ sensed by the laser range finder are firstly aggregated into a local fuzzy map $M_{o}(t+1)$ in polar coordinates and transformed into rectangular coordinate system, denoted as $\left.M_{o}(t+1)\right|_{(x, y)}$. The prediction algorithm of (8) and (9) is then used to crop from this local fuzzy map $\left.M_{o}(t)\right|_{(x, y)}$ and the last local fuzzy map $\left.M_{o}(t+1)\right|_{(x, y)}$ to generate two fuzzy maps, $\left.\widehat{M}_{o}(t+1)\right|_{(x, y)}$ and $\left.\widehat{M}_{o}(t)\right|_{(x, y)}$, for registration by the Cuckoo search [15], where a cost function of overlap between them is maximized. The registration results in a homogeneous transformation matrix ${ }_{t+1}^{t} T$ to be used for the local fuzzy map $M_{o}(t+1)$ to be integrated into the global fuzzy map in real time. The procedure iterates with the movement of the robot and the receiving of sensed data.

Figure 9 illustrates a situation of two cropped local fuzzy maps, $\left.\widehat{M}_{o}(t+1)\right|_{(x, y)}$ and $\left.\widehat{M}_{o}(t)\right|_{(x, y)}$, for registration. After successful registration by the Cuckoo search, an optimal transformation matrix ${ }_{t+1}^{t} T$ is obtained that has maximum overlapping for these two fuzzy maps, as shown in Figure 10.

\section{Experimental Results}

A moving robot of three omniwheels (or poly wheels) is built for experimental study; the robot is equipped with a Hokuyo URG-04LX laser range finder and a notebook of Intel Core i5 and 4 GB RAM, as shown in Figure 11.

The proposed procedure was successfully implemented to generate a global fuzzy map shown in Figure 12. In addition to the corridor, a closed passage is selected for robustness evaluation of the approach. The generated global fuzzy map is demonstrated in Figure 13 showing that the proposed method 


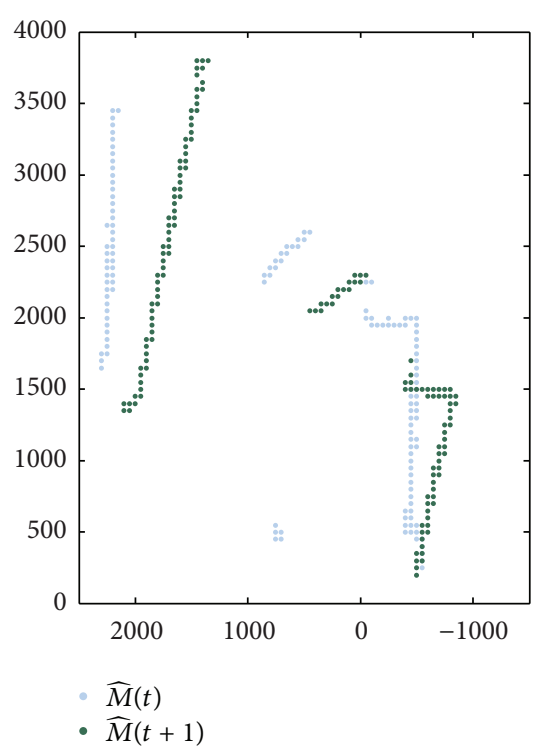

(a)

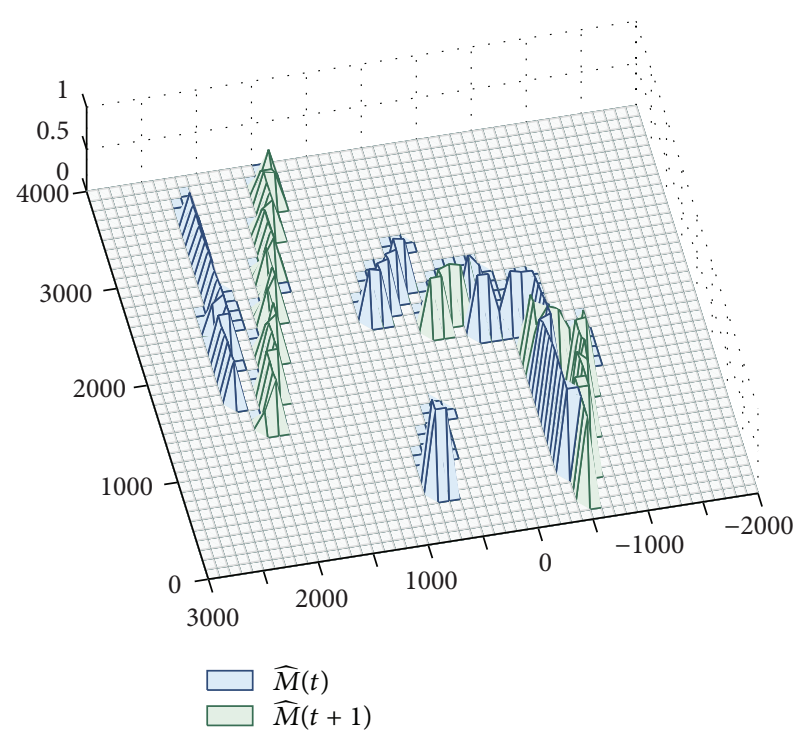

(b)

Figure 9: Two local fuzzy maps before registration. (a) 2D Fuzzy maps. (b) 3D fuzzy maps.

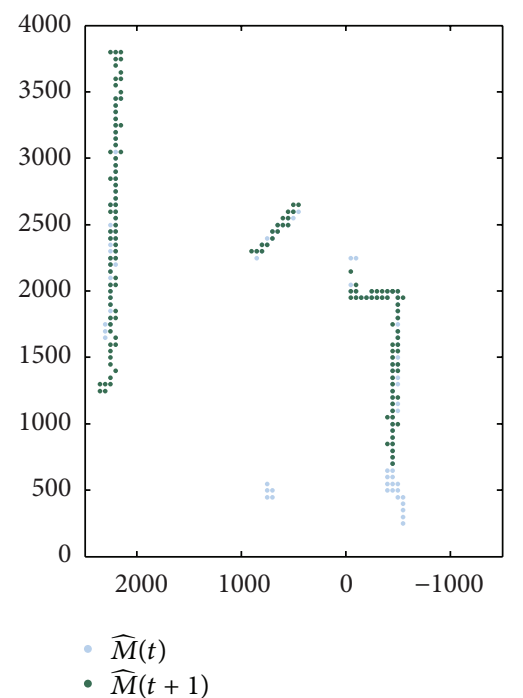

(a)

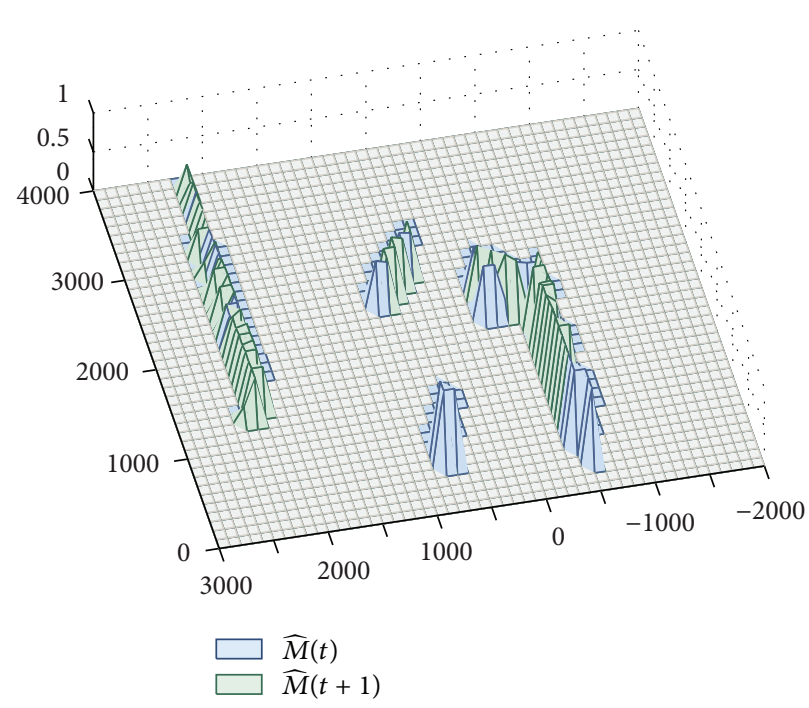

(b)

FIGURE 10: Two local fuzzy maps after registration showing maximum overlapping when compared with Figure 9. (a) 2D fuzzy maps. (b) 3D fuzzy maps.

is effective in generating a comprehensible and useful fuzzy map of the environment by the introduction of confidence fuzzy model and the use of the laser range finder.

\section{Conclusion}

This study proposes an effective SLAM algorithm using fuzzy confidence functions of the laser range finder. The fuzzy confidence functions are functions of both range and anger readings of the sensor which explicitly take sensor uncertainty and finite resolution into consideration. Based on the fuzzy functions, we are able to aggregate sensed data into local fuzzy maps by fuzzy union.

In aligning local fuzzy maps into a global fuzzy map, we developed a prediction strategy to crop the most potential part to be overlapped with global fuzzy map from the sensed local fuzzy maps. This strategy is experimentally evaluated to be effective in finding homogeneous transformation matrices by the Cuckoo search in real time.

In addition to the occupied obstacles, confidence fuzzy functions for available space are also implemented. The integration of occupied obstacles and available space allows us to 


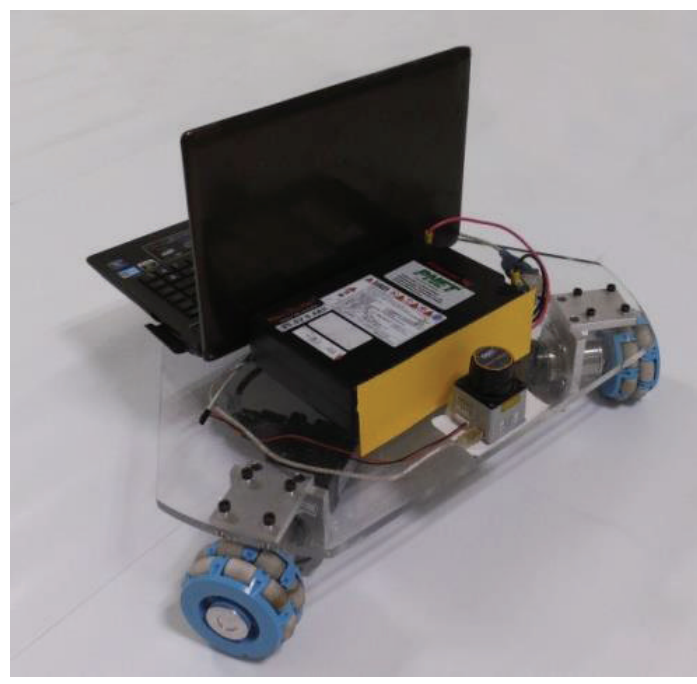

(a)

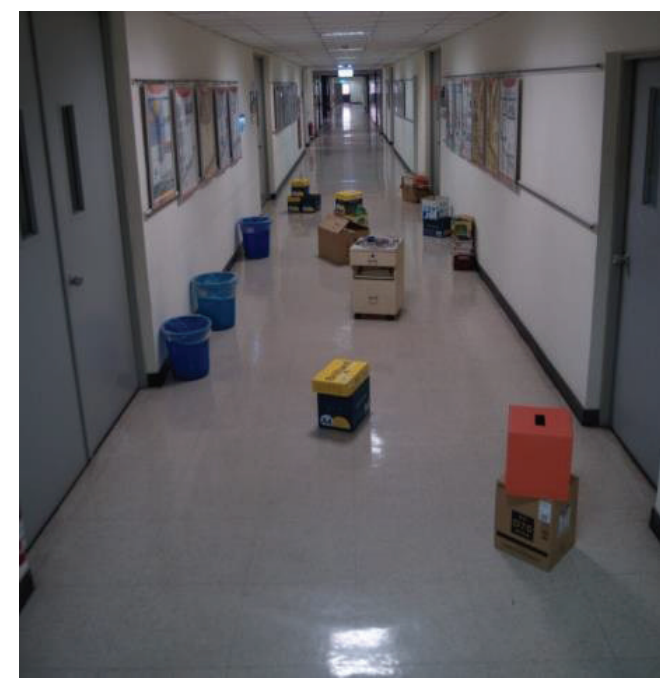

(b)

FIgURE 11: Experimental setup. (a) A moving robot equipped with three omniwheels. (b) A scenario of a corridor with obstacles along the passage.

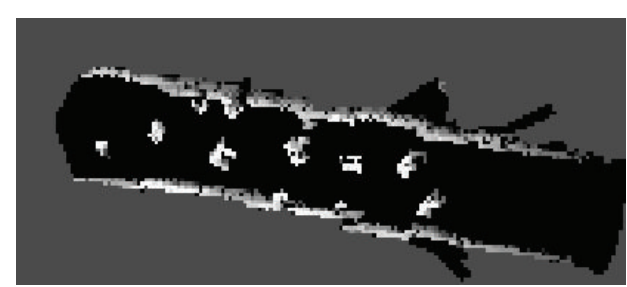

(a)

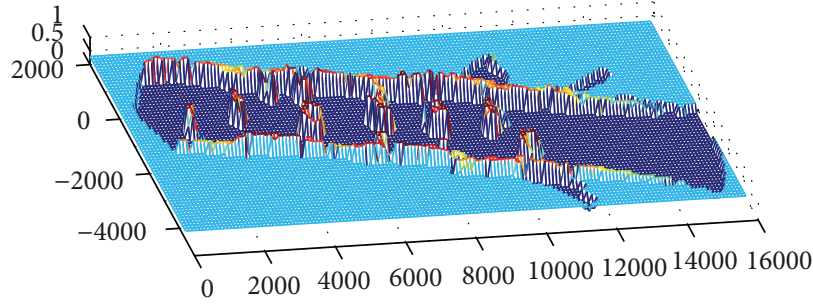

(b)

FIGURE 12: A global fuzzy map generated with the arrangement of Figure 11. (a) 2D fuzzy map. (b) 3D fuzzy map.

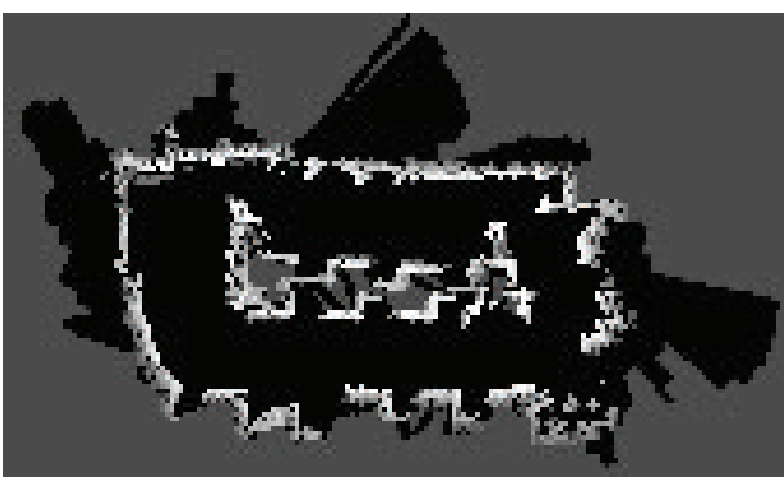

(a)

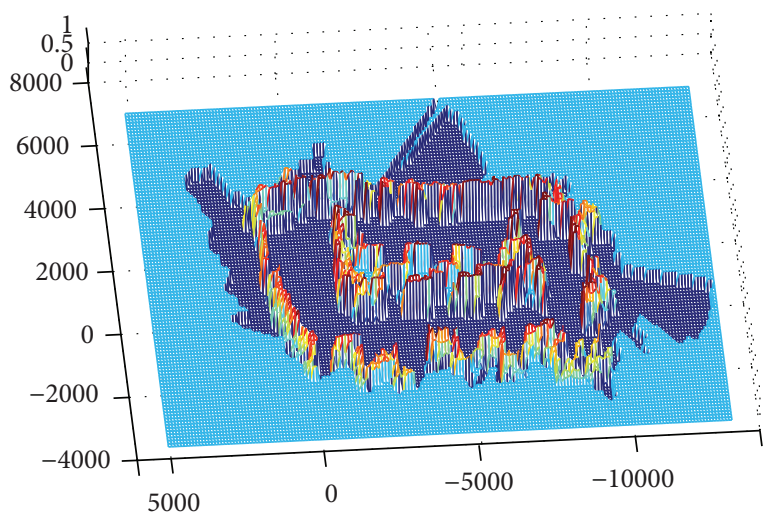

(b)

FIGURE 13: A comprehensive global fuzzy map of a closed passage generated by the proposed approach. (a) 2D fuzzy map. (b) 3D fuzzy map.

generate a comprehensive global fuzzy map that is suitable for both human comprehension and path design. Performance of the proposed architecture is verified by experiment results of a real-time mobile vehicle.
Indeed, fuzzy path planning seems to be more amenable to the proposed fuzzy map than its nonfuzzy counterparts. Further research aimed at exploiting the benefits of fuzzy maps to include measurement errors and uncertainty as 
an integral part of a map to improve reliability and robustness is needed to elucidate their role in navigation.

\section{Conflict of Interests}

The authors declare that there is no conflict of interests regarding the publication of this paper.

\section{Acknowledgments}

The authors are enormously grateful for the support from the National Science Foundation, Taiwan, under Contract nos. NSC 100-2221-E-182-008, NSC 101-2221-E-182-006, and NSC 102-2221-E-182-073; Chang Gung University and Chang Gung Memorial Hospital, Taiwan, under Contract nos. CMRPD1C0021, CMRPD2C0051, and CMRPD2C0052.

\section{References}

[1] H. Durrant-Whyte and T. Bailey, "Simultaneous localization and mapping: part I," IEEE Robotics and Automation Magazine, vol. 13, no. 2, pp. 99-108, 2006.

[2] T. Bailey and H. Durrant-Whyte, "Simultaneous localization and mapping (SLAM): part II," IEEE Robotics and Automation Magazine, vol. 13, no. 3, pp. 108-117, 2006.

[3] T. Sebastian, Exploring Artificial Intelligence in the New Millennium, Morgan Kaufmann, San Francisco, Calif, USA, 2003.

[4] S. Thrun, W. Burgard, and D. Fox, Probabilistic Robotics, The MIT Press, Cambridge, Mass, USA, 2005.

[5] K. S. Chong and L. Kleeman, "Mobile robot map building from an advanced sonar array and accurate odometry," Technical Report MECSE, 1996.

[6] M. A. K. Jaradat and R. Langari, "Line map construction using a mobile robot with a sonar sensor," in Proceedings of the IEEE/ASME International Conference on Advanced Intelligent Mechatronics (AIM '05), pp. 1251-1256, Monterey, Calif, USA, July 2005.

[7] J. Guivant, E. Nebot, and S. Baiker, "Autonomous navigation and map building using laser range sensors in outdoor applications," Journal of Robotic System, vol. 17, no. 10, pp. 565-583, 2000.

[8] A. J. Davison and N. Kita, "3D simultaneous localisation and map-building using active vision for a robot moving on undulating terrain," in Proceedings of the IEEE Computer Society Conference on Computer Vision and Pattern Recognition, pp. I384-I391, Kauai, Hawaii, USA, December 2001.

[9] M. Tomono, "3-D localization and mapping using a single camera based on structure-from-motion with automatic baseline selection," in Proceedings of the IEEE International Conference on Robotics and Automation, pp. 3342-3347, Barcelona, Spain, April 2005.

[10] S. Guadarrama and A. Ruiz-Mayor, "Approximate robotic mapping from sonar data by modeling perceptions with antonyms," Information Sciences, vol. 180, no. 21, pp. 4164-4188, 2010.

[11] S. Noykov and C. Roumenin, "Occupancy grids building by sonar and mobile robot," Robotics and Autonomous Systems, vol. 55, no. 2, pp. 162-175, 2007.

[12] A. Diosi and L. Kleeman, "Fast laser scan matching using polar coordinates," International Journal of Robotics Research, vol. 26, no. 10, pp. 1125-1153, 2007.
[13] A. J. Davison, I. D. Reid, N. D. Molton, and O. Stasse, "MonoSLAM: real-time single camera SLAM," IEEE Transactions on Pattern Analysis and Machine Intelligence, vol. 29, no. 6, pp. 1052-1067, 2007.

[14] G. Oriolo, G. Ulivi, and M. Vendittelli, "Fuzzy maps: a new tool for mobile robot perception and planning," Journal of Robotic Systems, vol. 14, no. 3, pp. 179-197, 1997.

[15] X.-S. Yang and S. Deb, "Cuckoo search via Lévy flights", in Proceedings of the World Congress on Nature and Biologically Inspired Computing (NABIC '09), pp. 210-214, Coimbatore, India, December 2009.

[16] X.-S. Yang, "Bat algorithm and cuckoo search: a tutorial," Studies in Computational Intelligence, vol. 427, pp. 421-434, 2013.

[17] K.-H. Lee and R. Ehsani, "Comparison of two 2D laser scanners for sensing object distances, shapes, and surface patterns," Computers and Electronics in Agriculture, vol. 60, no. 2, pp. 250262, 2008.

[18] R. Bréda, "Experimental measurement of parameters of the spatial scanner Hokuyo URG-04LX," Przeglad Elektrotechniczny, vol. 88, no. 5, pp. 132-135, 2012. 


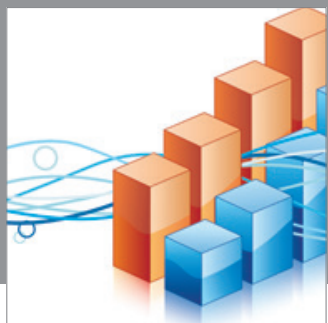

Advances in

Operations Research

mansans

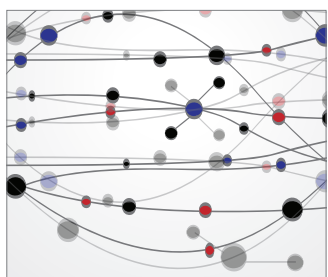

The Scientific World Journal
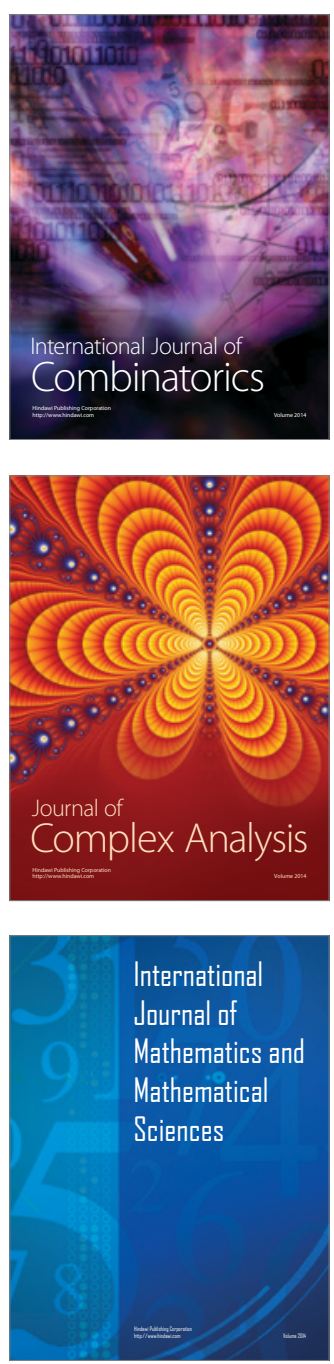
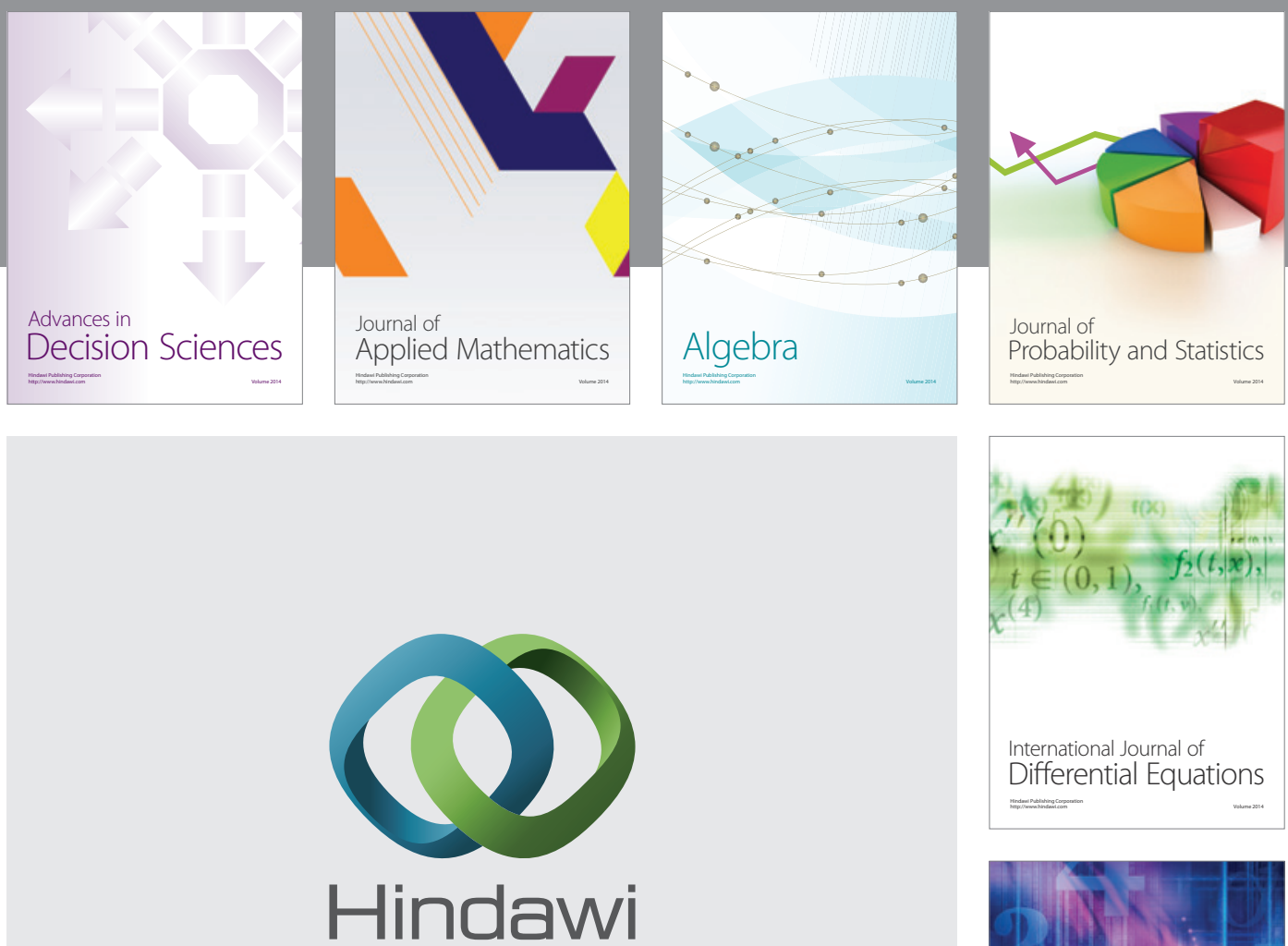

Submit your manuscripts at http://www.hindawi.com
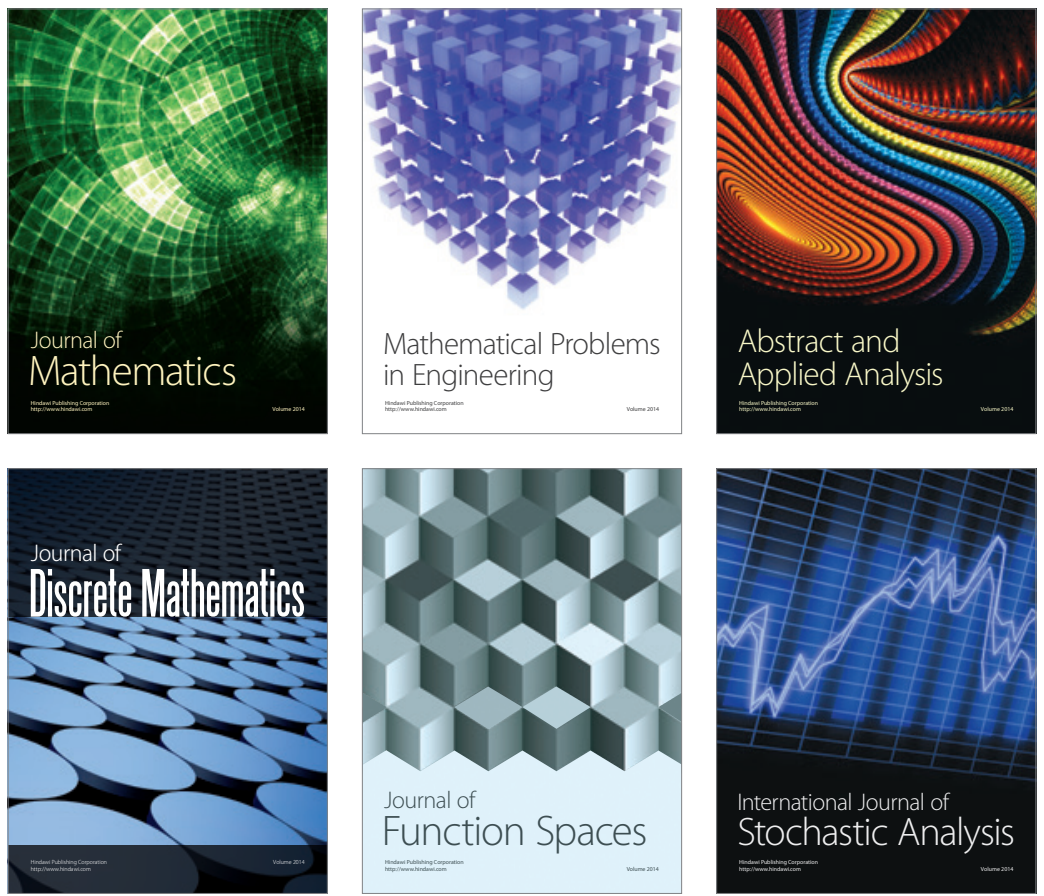

Journal of

Function Spaces

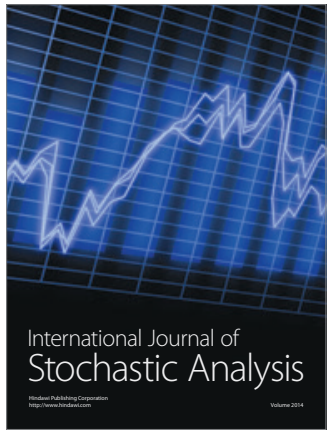

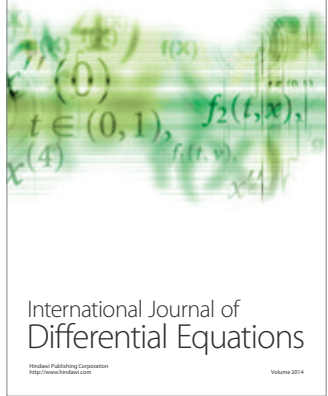
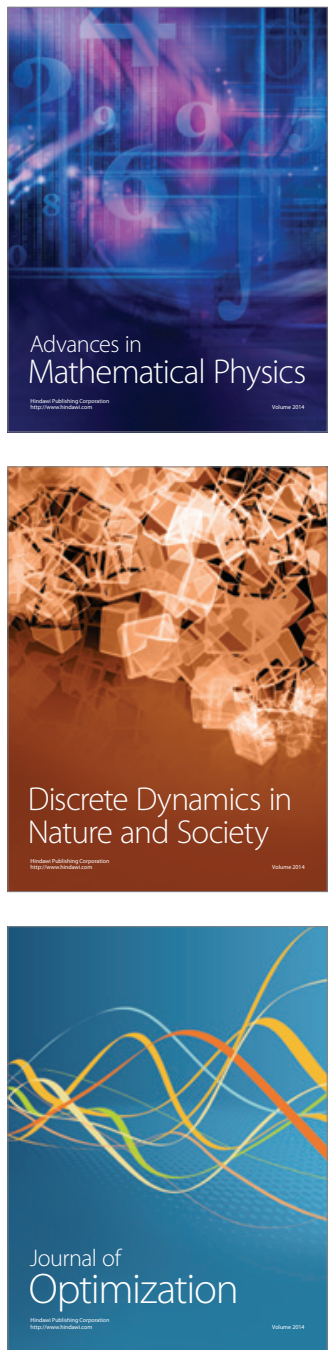